\title{
14
}

\section{A Practical Conference Key Distribution System}

\author{
Vassilis Chrissikopoulos and Dimitrios Peppes \\ Department of Informatics, University of Piraeus, \\ 80 Karaoli \& Dimitriou Str. 18534 Piraeus, Greece \\ e-mail: chris@cc.unipi.gr pepes@cc.unipi.gr
}

\begin{abstract}
We consider a variant of the Burmester-Desmedt conference key distribution system proposed at Eurocrypt '94. Our system is also based on public keys and has a low communication and computation complexity. Each party exchanges only three messages, and only a few multiplications are required. The security is based on the intractability of the Diffie-Hellman problem.
\end{abstract}

\section{INTRODUCTION}

Secure key distribution is a major issue in an open computer network environment, where data is readily available through distributed processing and distributed data bases. Many twoparty key distribution systems based on public keys are described in the literature (e.g. [1, $2,3,4,5,6,7,8,9,10,11,12,13])$. These employ a key exchange protocol in which a common session key is generated using randomness, to prevent replays, and secret information, for identification. If more than two parties are involved then a conference key distribution system is used. Designing such systems can be quite challenging because of the large number of interactions between the many users. Many conference key distribution systems have been proposed $[14,2,6,4,15,16]$. These however are either impractical, or only heuristic arguments are used to address their security. Our goal in this paper is to present a practical and secure conference key distribution system. Our system is based on the protocol presented in [17] and extends the two-party system proposed in $[1,11]$. Its security is based on the intractability of the Diffie-Hellman [18] problem,

The D-H problem: given integers $p, \alpha, X, Y$, find $X^{\log _{\alpha} Y} \bmod p$, if is exists.

This problem is generally assumed to be hard, even if the factorization of the order of $\alpha$ is known (cf. $[19,20]$ ). It is well known $[21,22,23]$ that this problem is easy if the Discrete Logarithm problem is easy then (but the converse may not be true). 
This paper is organized as follows. In the following section we present our protocol. Then, in Sections 3,4 we discuss its security: first against attacks by passive adversaries, and then against active adversaries.

\section{THE CONFERENCE KEY DISTRIBUTION SYSTEM}

We use a discrete logarithm setting with prime modulus $p$. The security parameter is $|p|$, the binary length of $p$. The basis is $\alpha$, whose order $q$ is large [24] (superpolynomial in $|p|)$. A Trusted Center chooses both $p$ and $\alpha$ and publishes these, together with $q$. Each user $U_{i}$ selects a secret key $s_{i} \in Z_{q}$, and then registers $P_{i}=\alpha^{s_{i}} \bmod p$ with the Trusted Centre as its public key.

\section{The protocol}

Let $U_{1}, U_{2}, \ldots, U_{n}$ be a dynamic set of users who want to generate a common conference key.

Step 1 Each user $U_{i}, i=1,2, \ldots, n$ selects uniformly at random $r_{i} \in Z_{q}$ and computes

$$
X_{i}=\alpha^{r_{i}} \bmod p .
$$

Then $U_{i}$ sends $X_{i}$ to the other parties in the conference.

Step 2 Each user $U_{i}, i=1,2, \ldots, n$ computes

$$
Y_{i} \equiv\left(P_{i+1}\right)^{r_{i}} /\left(X_{i-1}\right)^{s_{i}} \equiv\left(X_{i}\right)^{s_{i+1}} /\left(P_{i}\right)^{r_{i-1}}(\operatorname{modp})
$$

where the indices are taken in a cycle. Then $U_{i}$ sends $Y_{i}$ to the other parties in the conference.

\section{The Session Key}

The session key is,

$$
K \equiv \alpha^{r_{1} \mathbf{s}_{2}+r_{2} \mathbf{s}_{3}+\ldots+r_{2} \mathbf{s}_{1}}(\bmod p)
$$

which each user $U_{i}$ in the conference can compute since,

$$
K \equiv K_{i} \equiv\left(X_{i-1}\right)^{n s_{i}} \cdot Y_{i}^{n-1} \cdot Y_{i+1}^{n-2} \cdots Y_{i-2}(\bmod p)
$$


Figure 1 shows the conference key distribution system for three users.

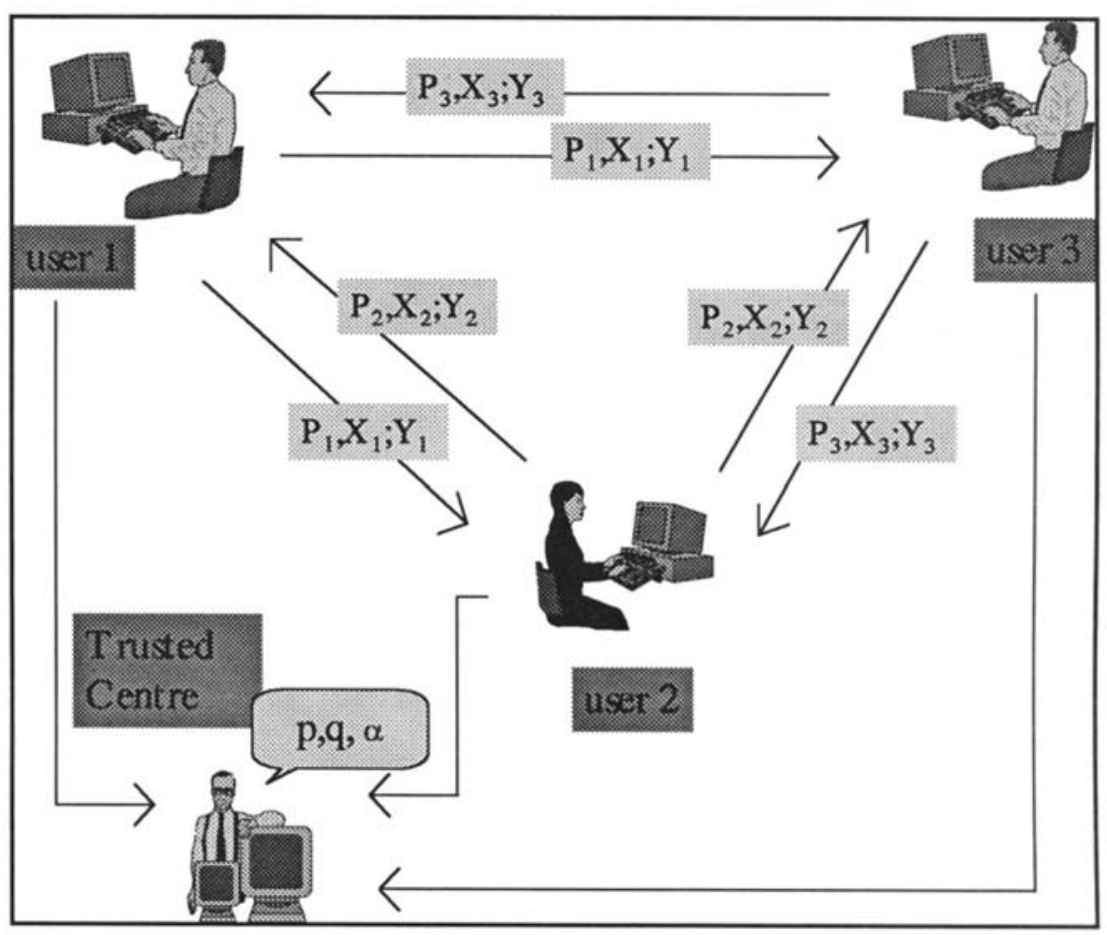

Figure 1. The Conference Key Distribution System for three users

Remark. This protocol is a modification of a protocol presented in [17]. When $n=2$ we have $\mathrm{Y}_{1} \equiv \mathrm{Y}_{2}^{-1} \equiv \mathrm{P}_{2}^{\mathrm{r}_{1}} / \mathrm{P}_{1}^{\mathrm{r}_{2}}(\bmod \mathrm{p})$

which both parties can compute, and therefore there is no need to exchange $Y_{1}, Y_{2}$. In this case

$\mathrm{K}=\alpha^{\mathrm{r}_{1} \mathbf{s}_{2}+\mathrm{r}_{2} \mathbf{s}_{1}} \operatorname{modp}$,

and we get the 'non-paradoxical' key distribution system proposed by Yacobi [11]. 


\section{RESILIENCE TO ATTACKS FROM PASSIVE ADVERSARIES}

We first consider the resilience of our system to attacks from a passive adversary, i.e. a passive eavesdropper.

Theorem. If the size of the conference is polynomially bounded in the length of $p$, and if the Diffie-Hellman problem is intractable, then the conference key distribution system described above is resilient to attacks from passive eavesdroppers.

Proof. This is similar to proof of Theorem 1 in [17]. Let $\mathrm{E}$ be a passive eavesdropper who succeeds with non-negligible probability in computing the key

$K_{1}=\left(X_{n}\right)^{n s_{1}} \cdot Y_{1}^{n-1} \ldots Y_{n-1}(\bmod p)$

of $U_{i}$. Fix $X_{n}, P_{1}$. Then select numbers $b_{2}, b_{3}, \ldots, b_{n}$ and $d_{2}, d_{3}, \ldots, d_{n}$, at random from $Z_{q}$, and set:

$X_{1} \equiv X_{n} \cdot \alpha^{-b} \cdot(\bmod p), X_{2} \equiv X_{1} \cdot \alpha^{b_{2}}(\bmod p), \ldots, X_{n-1} \equiv X_{1} \cdot \alpha^{b_{*-1}}(\bmod p)$

$P_{2} \equiv P_{1} \cdot \alpha^{d_{2}}(\bmod p), P_{3} \equiv P_{1} \cdot \alpha^{d_{3}}(\bmod p), \ldots, P_{n} \equiv P_{1} \cdot \alpha^{d} \cdot(\bmod p)$

Since

$Y_{i} \equiv\left(P_{i+1}\right)^{r_{i}} /\left(X_{i-1}\right)^{s_{i}} \equiv\left(P_{1} \cdot \alpha^{d_{i+1}}\right)^{r_{i}} /\left(X_{1} \cdot \alpha^{b_{i-1}}\right)^{s_{i}} \equiv\left(X_{i}\right)^{d_{i+1}} \cdot\left(P_{i}\right)^{-b_{i-1}}(\operatorname{modp})$,

with $b_{1}=d_{1}=0$, it is feasible to compute all the $Y_{i}$. So we have the necessary input for $E$. Then $E$ will succeed with non-negligible probability in computing $K_{1}$, and thus

$\left(X_{n}\right)^{n s_{1}} \bmod p$

from which it is feasible to compute

$\left(X_{n}\right)^{s_{1}} \equiv X_{n}^{\log _{a} P_{1}} \bmod p$,

since it is feasible to extract $n$-th residues in $Z_{p}$ when $n$ is polynomially bounded $[25,26]$. Hence $\mathrm{E}$ can compute

$X_{n}^{\log _{\alpha} P_{1}} \bmod p$

from $X_{n}$ and $P_{1}$, which violates the intractability of the Diffie-Hellman problem. 


\section{RESILIENCE TO ATTACKS FROM ACTIVE ADVERSARIES}

We consider a very powerful adversary $\mathrm{E}$ who can read the calls which the parties exchange, modify the calls, replay old calls, and conduct sessions in parallel with many parties 'playing one against the other' [27,28]. Furthermore the adversary has access to some (chosen) old session keys. However we shall assume that the adversary is polynomially bounded.

\subsection{The protocol leaks knowledge}

Burmester and Desmedt point out in [29], that the two-party system $(n=2)$ leaks knowledge under a general attack. This knowledge can be used by an adversary to compute a session key if the adversary has access to some session keys[30]. We shall now describe such an attack for the case $n=3$ based on the 'triangle attack' in [30].

\section{A known-key attack}

Suppose that $U_{1}, U_{2}, U_{3}$ have computed $K \equiv \alpha^{r_{1} s_{2}+r_{2} s_{3}+r_{3} s_{1}}(\bmod p)$, with calls $X_{1}, X_{2}, X_{3}$. The adversary $\tilde{U}_{4}$ is an insider with secret key $s_{4}$, and public key $P_{4}$, who wants to compute the key $\mathrm{K}$. The adversary $\tilde{U}_{4}$ gets $\mathrm{X}_{1}, \mathrm{X}_{2}, \mathrm{X}_{3}$ by eavesdropping. To compute $\mathrm{K}, \tilde{U}_{4}$ first gets the partial key

$\mathrm{k}_{1}=\alpha^{\mathrm{r}_{3} \mathrm{~s}_{1}} \bmod \mathrm{p}$

from the session key

$K_{1}=\alpha^{r_{1}^{\prime} s_{4}+r_{3} s_{1}} \bmod p$

of the two-party protocol $\left(U_{1}, \tilde{U}_{4}\right)$ with calls $X_{1}^{\prime}=\alpha^{r_{1}^{\prime}} \bmod p, X_{3}$. The adversary $\tilde{U}_{4}$ can compute $k_{1}$ because she knows $s_{4}$. Similarly $\tilde{U}_{4}$ can get the partial key

$\mathbf{k}_{2}=\alpha^{\mathrm{r}_{1} \mathrm{~s}_{2}} \bmod \mathbf{p}$

from the session key

$\mathrm{K}_{2}=\alpha^{\mathrm{r}_{2}^{\prime} \mathrm{s}_{4}+\mathrm{r}_{1} \mathrm{~s}_{2}} \bmod \mathrm{p}$

of the protocol $\left(U_{2}, \tilde{U}_{4}\right)$ with calls $X_{2}^{\prime}=\alpha^{r_{2}^{\prime}} \bmod p, X_{1}$, and

$k_{3}=\alpha^{r_{2} s_{3}} \bmod p$ 
from the session key

$\mathrm{K}_{3}=\alpha^{\mathrm{r}_{3}^{\prime} \mathrm{s}_{4}+\mathrm{r}_{2} \mathrm{~s}_{3}} \bmod \mathrm{p}$

of the protocol $\left(U_{3}, \tilde{U}_{4}\right)$ with calls $X_{3}^{\prime}=\alpha^{r_{3}^{\prime}} \bmod p, X_{2}$. By multiplying $k_{1}, k_{2}, k_{3}, \tilde{U}_{4}$ then gets $\mathrm{K}$. Observe that with known-key attacks the adversary has access to some chosen session keys.

Different ways to prevent such attacks are described in [30]. One such way is for the users to take as session key $h a s h(K)$, where hash is an appropriate hash function, instead of $\mathrm{K}$ itself. This will prevent this attack. However the messages $Y_{i}$ sent in Step 2 of the protocol also leak knowledge which an adversary can use to compute earlier session keys. We now describe such an attack.

\section{Another insider attack}

Observe that

$Y_{i}=\alpha^{r_{i} s_{i+1}-r_{i-1} s_{i}} \bmod p$

is the session key of the two-party protocol $\left(U_{i}, U_{i+1}\right)$ with calls $X_{i}, X_{i-1}^{-1}$. The messages $Y_{i}$ cannot be hashed as in the previous case because they are needed to compute the session key $K$. If the adversary $\tilde{U}_{i+1}=U_{i+1}$ uses the inverse of an earlier call $X_{i-1}$, then $\tilde{U}_{i+1}$ can compute the partial key

$k_{i}=\alpha^{r_{i-1} s_{i}} \bmod p$.

So by arranging sessions with the other parties, one at a time, the adversary can compute all the factors of $\mathrm{K}$, and hence $\mathrm{K}$ itself. To prevent this, the calls $\mathrm{X}_{\mathrm{i}}$ must be authenticated. This would make it impossible for the adversary to re-use earlier calls in her attacks. Different ways of doing this are described in [17]. Here we propose that each party $U_{i}$ in Step 1 of the protocol proves knowledge proof of the discrete logarithm $r_{i}$ of $X_{i}$. This can be done by using a zero-knowledge proof of the discrete logarithm (e.g. [31]). For practicality we propose that a signature scheme based on zero-knowledge proofs is used, as in [32]. This means that each $U_{i}$ in Step 1 of the protocol needs only send one more message, the signature of the conference key string $P_{1}, \ldots, P_{n}$ with key $X_{i}$.

We now address the general problem of security. With the modified protocol the messages that the adversary may use must be independent of those sent in the earlier sessions. This means that the 'history' of the adversary which consists of messages exchanged earlier, can be simulated. This scenario is analogous to the one encountered when dealing with dishonest provers $\tilde{\mathrm{P}}$ in interactive zero-knowledge proofs systems of knowledge [33]. These systems 
make it possible for a prover $\mathrm{P}$ to prove to a verifier $\mathrm{V}$ that it knows some secret information without leaking any knowledge about it to $\mathrm{V}$. If any other prover $\tilde{\mathrm{P}}$, possibly dishonest, succeeds in convincing $\mathrm{V}$ with non-negligible probability that it knows P's secret, then $\tilde{\mathrm{P}}$ must know the secret, or be able to compute it (the soundness condition [33]). $\tilde{\mathbf{P}}$ is allowed to collaborate with a dishonest verifier $\tilde{V}$ who may interact with the honest prover $P$ (in an attempt to learn from $P$ something about the secret). The knowledge of $\tilde{\mathrm{P}}$ is checked by an 'extractor' who can obtain the secret from $\tilde{\mathbf{P}}$ by 'probing and resetting' $\tilde{\mathbf{P}}$. To make the link with our scenario we take the adversary $\mathrm{E}$ to be both $\tilde{\mathrm{P}}$ and $\tilde{\mathrm{V}}$, and the 'broken' secret key to be the secret of $P$. In the full paper we will show that the techniques of zero-knowledge proofs can be used to prove that our modified protocol (with signatures) is resilient from active adversaries, provided the Diffie-Hellman problem is intractable.

\section{Acknowledgment}

The authors would like to thank Mike Burmester and Yvo Desmedt for many helpful discussions and in particular for pointing out the weaknesses in our protocol.

\section{REFERENCES}

1. T. Matsumoto, Y. Takashima and H. Imai. On Seeking Smart Public Key Distribution Systems. The Transactions of the IECE of Japan, Vol. E69(2), 1986, pp. 99-106.

2. K. Koyama and $\mathrm{K}$. Ohta. Identity-based conference key distribution systems. In C. Pomerance (ed.), Advances in Cryptology-Cypto' 87, Lecture Notes in Computer Science 293, Springer-Verlag, 1988, pp. 175-185.

3. E. Okamoto. Key distribution systems based on identification information. In C. Pomerance (ed.), Advances in Cryptology-Cypto' 87, Lecture Notes in Computer Science 293, Springer-Verlag, 1988, pp. 194-202.

4. S. Tsujii and T. Itoh. An ID-based cryptosystem based on the discrete logarithm. IEEE $J$. Selected Areas Commun., SAC-8, 1989, pp. 467-473.

5. J. Brandt, I. Damgard, P. Landrock and T. Pedersen. Zero-knowledge authentication scheme with secret key exchange. In S. Goldwasser (ed.), Advances in CryptologyCypto'88, Lecture Notes in Computer Science 403, Springer-Verlag, 1989, pp. 583-588.

6. E. Okamoto and K. Tanaka. Key distribution system based on identification information. IEEE J. Selected Areas Commun., SAC-7, 1989, pp. 481-485.

7. F. Bauspiess and H.J. Knobloch. How to Keep Authenticity Alive in a Computer Network. In J.J. Quisquater and J. Vandewalle (eds.), Advances in Cryptology-Eurocrypt' 89 , Lecture Notes in Computer Science 434, Springer-Verlag, 1990, pp. 36-46.

8. Y. Yacobi and Z. Shmuely. On key distribution systems. In G. Brassard (ed.), Advances in Cryptology-Cypto' 89, Lecture Notes in Computer Science 435, Springer-Verlag, Berlin 1990, pp. 344-355.

9. C.G. Gunther. An identity-based key exchange protocol. In J.J. Quisquater and J. Vandewalle (eds.), Advances in Cryptology-Eurocypt' 89, Lecture Notes in Computer Science 434, Springer-Verlag, 1990, pp. 29-37

10. T. Beth and H.J. Knobloch. Open network authentication without online server. Proc. Symposium on Computer Security, CS 90, Rome 1990, 1991, pp. 160-165. 
11. Y. Yacobi. A key Distribution Paradox. In A.J. Menezes and S.A. Vanstone (eds.), Advances in Cryptology-Cypto' 90, Lecture Notes in Computer Science 537, SpringerVerlag, Berlin 1991, pp. 268-273.

12. R. Bird, I Gopal, A. Herzberg, P. Jansen, S. Kutten, R. Molva and M. Yung. Systematic design of two-party authentication protocols. In J. Feigenbaum (ed.), Advances in Cryptology-Crypto' 91, Lecture Notes in Computer Science 576, Springer-Verlag, 1992, pp. 44-61.

13. M. Bellare and P. Rogaway. Entity authentication and Key Distribution. in D.R. Stinson (ed.), Advances in Cryptology-Crypto' 93, Lecture Notes in Computer Science 773, Springer-Verlag, 1994, pp. 232-249.

14. I. Ingemarsson, D.T. Tang and C.K. Wong. A conference key distribution system. IEEE Trans. Inform. Theory, 28 , 1982, pp. 714-720.

15. M.J. Fischer and R.N.Wright. Multiparty secret key exchange using a random deal of cards. In J. Feigenbaum (ed.), Advances in Cryptology-Cypto' 91, Lecture Notes in Computer Science 576, Springer-Verlag, 1992, pp. 141-155.

16. C. Blundo, A. De Santis, A. Herzberg, S. Kutten, U. Vaccaro, M. Yung. Perfectly-secure key distribution for dynamic conferences. In E. Brickell (ed.), Advances in CryptologyCypto' 92, Lecture Notes in Computer Science 740, Springer-Verlag, 1993, pp. 471-487.

17. M. Burmester and Y. Desmedt. A Secure and Efficient Conference Key Distribution System. To appear in the Proceedings of Eurocrypt ' 94.

18. W. Diffie and M.E. Hellman. New directions in cryptography. IEEE Trans. Inform. Theory, IT-22, 1976, pp. 644-654.

19. M. Blum and S. Micali. How to generate cryptographically strong sequences of pseudorandom bits. Siam J. Comput., 13(4), 1984, pp. 850-864.

20. G. Brassard, D. Chaum, and C.Crepeau. Minimum disclosure proofs of knowledge. Journal of Computer and Systems Sciences, 37 (2), 1988, pp. 156-189.

21. A.M. Odlyzko. Discrete logs in a finite field and their cryptographic significance. In N. Cot T. Beth and Ingemarsson, (ed.), Advances in Cryptology, Proc. of Eurocrypt 84, Lecture Notes in Computer Science 209, Springer-Verlag, 1984, pp. 224-314.

22. D. Coppersmith, A. Odlyzko, and R. Schroeppel. Discrete logarithms in GF(p). Algorithmica, 1986, pp. 1-15.

23. A. Menezes, S. Vanstone and T. Okamoto. Reducing elliptic curve logarithms to logarithms in a finite field. In Proceedings of the Twenty Third annual ACM Symp. Theory of Computing, STOC, 1991, pp. 80-89.

24. C.P. Schnorr. Efficient identification and signatures for smart cards. Journal of Cryptology, 4(2), 1991, pp. 161-174.

25. E.R. Berlekamp. Factoring polynomials over large finite fields. Mathematics of Computation, 24(111), 1970, pp. 713-735.

26. M. Rabin. Probabilistic Algorithms in Finite Fields. SIAM Journal on Computing, 9(2), 1980, pp. 273-280.

27. Y. Desmedt, C. Goutier and S. Bengio. Special uses and abuses of the Fiat-Shamir passport protocol. In C. Pomerance (ed.), Advances in Cryptology-Cypto' 87, Lecture Notes in Computer Science 293, Springer-Verlag, 1988, pp. 21-39.

28. S. Bengio, G. Brassard, Y.Desmedt, C. Goutier, and J.J. Quisquater, Secure implementations of identification systems. Journal of Cryptology, 4(2), 1991, pp. 175184. 
29. Y. Desmedt and M. Burmester. Towards Practical 'proven secure' authenticated key distribution. Proceedings 1st ACM Conference on Computer and Communication Security, Fairfax, Virginia, ACM Press 1993, pp. 228-231.

30. M. Burmester. On th Risk of Opening Distributed Keys. In E. Brickell (ed.), Advances in Cryptology-Cypto' 94, Lecture Notes in Computer Science 839, Springer-Verlag, 1994, pp. 308-317.

31. D. Chaum, J.-H. Evertse, J. van de Graaf and R. Peralta. Demonstrating possession of a discrete logarithm without revealing it. In Odlyzko (ed.), Advances in Cryptology-Crypto 86, Lecture Notes in Computer Science, 263, Springer-Verlag, Berlin 1987, pp. 200-212.

32. A. Fiat and A. Shamir. How to prove yourself: Practical solutions to identification and signature problems. In A. Odlyzko (ed.) Advances in Cryptology-Crypto 86, Lecture Notes in Computer Science, 263, Springer-Verlag, Berlin 1987, pp. 186-194

33. U. Feige, A. Fiat and A. Shamir. Zero knowledge proofs of identity. Journal of Cryptography, 1 (2), 1988, pp. 77-94. 